Théologiques

Théologiques

\title{
La bioéthique, trente ans plus tard : qu'en est-il des espoirs des fondateurs face à la recherche?
}

\section{Michel Bergeron}

Volume 7, numéro 1, printemps 1999

Les trente ans de la bioéthique

URI : https://id.erudit.org/iderudit/024972ar

DOI : https://doi.org/10.7202/024972ar

Aller au sommaire du numéro

\section{Éditeur(s)}

Faculté de théologie de l'Université de Montréal

\section{ISSN}

1188-7109 (imprimé)

1492-1413 (numérique)

Découvrir la revue

Citer cet article

Bergeron, M. (1999). La bioéthique, trente ans plus tard : qu'en est-il des espoirs des fondateurs face à la recherche? Théologiques, 7(1), 35-49.

https://doi.org/10.7202/024972ar
Résumé de l'article

Les questions à portée éthique qui se manifestent en recherche traversent la bioéthique de ses origines à ses développements les plus récents. Formulées initialement surtout de l'intérieur des frontières américaines, berceau de ce mouvement social, elles trouvent aujourd'hui écho dans plusieurs pays du monde, même en dehors du contexte occidental. Leur présence se fait particulièrement vive là où l'expérimentation porte sur des êtres humains. Depuis les années 1960, la bioéthique a évolué et s'est " spécialisée » en différents secteurs. Qu'est-il advenu de son volet " éthique de la recherche » entre les jours de ses premiers balbutiements et ceux qui ont vu la publication de l'Enoncé de politique des trois conseils? Dans quelle mesure les espoirs qui habitaient ses fondateurs se sont-ils réalisés? Ces deux questions feront l'objet de la présente réflexion. 


\title{
La bioéthique, trente ans plus tard : qu'en est-il des espoirs des fondateurs face à la recherche?
}

\author{
Michel BERGERON \\ Étudiant, Ph.D. en sciences \\ humaines appliquées (bioéthique) \\ Université de Montréal \\ et directeur, Service de la recherche \\ Université Saint-Paul
}

Les questions à portée éthique qui se manifestent en recherche, traversent la bioéthique de ses origines à ses développements les plus récents. Formulées initialement surtout de l'intérieur des frontières américaines, berceau de ce mouvement social, elles trouvent aujourd'hui écho dans plusieurs pays du monde, même en dehors du contexte occidental. Leur présence se fait particulièrement vive là où l'expérimentation porte sur des êtres humains. Depuis les années 1960, la bioéthique a évolué et s'est "spécialisée " en différents secteurs. Qu'est-il advenu de son volet "éthique de la recherche " entre les jours de ses premiers balbutiements et ceux qui ont vu la publication de l'Énoncé de politique des trois conseils ${ }^{1}$ ? Dans quelle mesure les espoirs qui habitaient ses fondateurs se sont-ils réalisés? Ces deux questions feront l'objet de la présente réflexion.

1 CONSEIL DE RECHERCHES MÉDICALES DU CANADA, CONSEIL DE RECHERCHES EN SCIENCES NATURELLES ET GÉNIE DU CANADA ET CONSEIL DE RECHERCHES EN SCIENCES HUMAINES DU CANADA, Énoncé de politique des trois conseils. Éthique de la recherche avec des êtres humains. Ottawa, Conseil de recherches médicales du Canada, août 1998. 
Trois étapes ponctueront notre parcours. Dans un premier temps, nous ferons une brève incursion du côté des racines historiques du mouvement bioéthique. Puis, nous examinerons certains faits et textes qui ont marqué la période initiale comprise entre la fin des années 1960 et le milieu des années $1970^{2}$. Cela nous permettra d'y saisir quelques espoirs des fondateurs. Enfin, trente ans après, nous déterminerons ce qu'il est advenu de ces espoirs à la lumière de la situation actuelle en éthique de la recherche.

\section{Bioéthique et recherche}

La bioéthique est enracinée profondément dans l'histoire du développement scientifique. Ses points d'ancrage sont fixés dans une série d'événements et de codes qui ont marqué la science et la médecine depuis l'Antiquité ${ }^{3}$. Nous nous contenterons ici d'en souligner quelques-uns, peut-être moins connus, mais que nous considérons importants pour la période comprise entre 1800 et 1960 .

En 1803, la nécessité de l'évaluation scientifique par les pairs fut énoncée 4 pour une première fois. Il faudra toutefois attendre près de 150 ans, soit en 1953, avant que les National Institutes of Health américaines $(\mathrm{NIH})$ en formalisent l'exercice par l'entremise des an-

2 H. DOUCET, Au pays de la bioéthique. L'éthique biomédicale aux ÉtatsUnis. Genève, Labor et Fides (Le champ éthique, 29), 1996, p. 34.

3 Voir, entre autres, T.L. BEAUCHAMP et J.F. CHILDRESS, Principles of Biomedical Ethics. New-York, Oxford University Press, 1979, à l'annexe II; N.S. JECKER, A.R. JONSEN et R.A. PEARLMAN, Bioethics. An Introduction to the History. Methods and Practice. Boston, Jones and Bartkett Publishers, 1997, 416 p.; A.R. JONSEN, "The Birth of Bioethics", dans Hastings Center Report, Special Supplement, November-December 1993, 4 p.; S.J. ReISER, A.J. DYCK et W.J. William, Ethics in Medicine. Historical Perspectives and Contemporary Concerns. Cambridge, Mass., The M.I.T. Press, 1977, 679 p.; D.J. RoTHMAN, Strangers at the Bedside. A History of How Law and Bioethics Transformed Medical Decision Making. New-York, Basic Books, 1991, 303 p.; M. SIEGLER, "Bioethics: A Critical Consideration », dans Église et théologie 13 (1982) 297-298.

4 R.J. LEVINE, Ethics and Regulation of Clinical Research. 2nd Edition, Baltimore-Munich, Urban \& Schwarzenberg, 1986, p. 33. 
cêtres des Institutional Review Boards (IRB) qu'étaient les Clinical Research Committees ${ }^{5}$. Entre temps, en 1865, Claude Bernard posa les prémices de la recherche clinique moderne ${ }^{6}$ et formula l'idée «que le principe de moralité médicale et chirurgicale consiste... à ne jamais pratiquer sur un homme une expérience qui ne pourrait que lui être nuisible à un degré quelconque, bien que le résultat pût intéresser beaucoup la science, c'est-à-dire la santé des autres ${ }^{7}$.» Puis, dès 1886, Charles Francis Withington parla de conflit possible entre les intérêts de la science médicale et ceux du patient comme individu ${ }^{8}$.

Durant cette même période, l'horizon éthique en général s'élargissait, et le concept de droit s'étendait, aux États-Unis, de l'émancipation des esclaves (1863) à la protection des espèces (1873) en passant par les droits des femmes (1920), des Amérindiens (1924), des travailleurs (1938) et "des Noirs" $(1957)^{9}$. Une telle progression ne fut toutefois pas unique à ce pays comme en font foi les Cahiers

5 La mise sur pied des Clinical Research Committees (CRC) fut exigée par le Bureau médical des NIH immédiatement après la création du Centre clinique en 1953. Pour être admis à ce centre, patients et volontaires devaient accepter d'être intégrés à un programme de recherche conduit par un des médecins des neuf instituts participants. Les patients y demeuraient vingt jours et des soins médicaux ne leur étaient fournis que dans le cadre du projet. Composés uniquement de scientifiques et de médecins, les CRC furent ainsi créés afin d'évaluer les projets cliniques impliquant un danger inhabituel. Leur évolution vers les IRB découla de la complexification et du manque de compréhension concernant le respect de l'évaluation des projets de recherche avec des sujets humains. (Tiré de M.B. LIPSETT, J.C. FLETCHER et M. SECUNDY, «Research Review at NIH ", dans Hastings Center Report, vol. 9, no. 1, Février 1979, p. 18.)

6 S.J. REISER et al., Ethics in Medicine, p. 255.

7 C. BERNARD, Introduction à l'étude de la médecine expérimentale. Paris, Librairie Ch. Delagrave, 1898, p. 160.

8 S.J. REISER et al., Ethics in Medicine, p. 260.

9 R.F. NASH The Rights of Nature. A History of Environmental Ethics. Madison, Wis., University of Wisconsin Press, 1989, p. 5-7; D.J. ROTHMAN, Strangers at the Bedside, p. 15. 
Laënnec ${ }^{10}$. En effet, dès 1936, en France, la Cour statuait qu'un véritable contrat se formait entre patient et médecin ${ }^{11}$. Puis, en 1945, des textes administratifs créèrent la Commission des Essais dont le mandat était d'approuver les dispositions convenues entre des compagnies et des experts concernant l'essai de produits pharmaceutiques ${ }^{12}$. Comme l'indiquent les Cahiers en avant-propos, ces développements furent basés sur la vision que "la Société a... le devoir d'organiser et de contrôler les interventions sur l'homme... Il faut que la conscience des médecins soit également avertie des excès toujours possibles en cette matière ${ }^{13}$. " Toutefois, malgré ces développements des deux côtés de l'Atlantique, les sources consultées ne lais sent entrevoir aucun lien entre la France et les États-Unis concernant la mise en place de mécanismes d'évaluation.

Toujours au cours de ce siècle et demi, des codes portant sur la recherche et les activités professionnelles voyaient le jour (Nuremberg en 1947, Helsinki en 1964 avec des révisions faites en $1975,1983$ et 1989 , etc. $)^{14}$ tandis que le développement des comités ayant pour rôle d'examiner les protocoles de recherche ${ }^{15}$ prenait de l'ampleur. Ainsi, ces codes, ces normes et ces structures en vinrent à constituer des cadres favorisant un exercice éthique de la responsabilité sociale des scientifiques selon trois niveaux hiérarchiques: celui du scientifique en tant qu'individu, celui des

10 CAHIERS LAËNNEC, L'expérimentation humaine en médecine. Paris, P. Lethielleux, 1952, $219 \mathrm{p}$.

11 P. TANRET, "Le médecin et l'expérimentation humaine" , dans L'expérimentation humaine en médecine. Cahiers Laënnec, Paris, P. Lethielleux, 1952, p. 48.

12 C. VAILLE, "Textes administratifs et expérimentation humaine", dans L'expérimentation humaine en médecine. Cahiers Laënnec, Paris, P. Lethielleux, 1952, p. 122.

13 CAHIERS LAËNNEC, L'expérimentation humaine, p. 11.

14 La plupart de ces codes sont déjà bien connus. Se référer à la note 3 pour les sources pertinentes.

15 M.B. LIPSETT et al., «Research Review at NIH », p. 18. 
sociétés et des institutions scientifiques ainsi que celui des gouvernements 16 .

Parallèlement à ce développement que, faut-il le noter, des scientifiques sensibles à la situation des sujets humains en recherche initièrent, se manifesta, entre 1965 et 1980, un mouvement public qui s'en prit à la collusion entre la science et le pouvoir. Les obligations de productivité de la recherche s'accrurent suivant en cela la croissance vertigineuse des fonds mis à la disposition des chercheurs. La société commença donc à exercer une forte pression sur ces derniers ${ }^{17}$. C'est dans ce contexte que trois événements significatifs pour la bioéthique vont se produire : 1) la parution de l'article "They Decide Who Lives", publié par le magazine Life du 9 novembre 1962 et portant sur le choix des patients à traiter dans le cas de ressources restreintes (ici un appareil à dialyse), 2) la publication de l'article de Henry Beecher (1966) portant sur vingt-deux cas de pratiques douteuses lors d'expérimentations et 3) la première transplantation cardiaque réussie par Christiaan Barnard (1967)18. Ces trois moments, pris individuellement ou ensemble, constituent, de l'avis même des "pionniers ${ }^{19}$ ", les instants marquant la naissance de ce mouvement social qu'est la bioéthique.

16 C'est à ces trois niveaux que van $\mathrm{R}$. Potter se réfère dans "Bioethics for Whom" des Annals of the New-York Academy of Sciences, vol. 196,1972 , p. 200.

17 Pour un regard plus en profondeur sur ce sujet voir A. FAGOTLARGEAULT, L'homme bio-éthique. Pour une déontologie de la recherche sur le vivant. Paris, Maloine S.A. Éditeur (Recherches interdisciplinaires), 1985, pp. 11-15 ainsi que G.H. KIEFFER, Bioethics: A Textbook of Issues. Don Mills, Ontario, Addison-Wesley Publishing Co., 1979, p. 240.

A.R. JONSEN et R.A. PEARLMAN, The Birth of Bioethics, p. S2.

19 Ce terme refère aux quelques soixante participants présents à la conférence intitulée The Birth of Bioethics qui s'est tenue les 23 et 24 septembre 1992 à l'Université de Washington à Seattle. Le comité organisateur avait alors défini comme "pionniers" les personnes dont le nom apparaissait dans la première édition de la Bibliography of Bioethics de 1975. Voir à ce sujet A.R. JONSEN et R.A. PEARLMAN, The Birth of Bioethics, p. S1. 
La référence à trois moments de la naissance de la bioéthique nous apparaît intéressante lorsque l'on tient compte de la subdivision qu'a connue ce mouvement au cours des années. L'article de Life oriente vers les questions touchant la rareté des ressources et la disponiblité des traitements. En ce sens, il peut être relié aux questions d'éthique organisationnelle en milieu de santé. De son côté, la transplantation cardiaque réussie par le Dr Barnard ouvrait à une série de questions en éthique médicale dont celle de l'intégrité de la personne. Il faut se rappeller ici que le cœur fut de tout temps consi déré comme le siège des sentiments et des valeurs par la croyance populaire. Enfin, l'article de Beecher nous oriente sans contredit vers l'éthique de la recherche.

Publié dans le New England Journal of Medicine sous le titre "Ethics and Clinical Research", l'article de Henry Beecher aura l'effet d'une bombe qui secouera sérieusement les milieux scientifiques et créera des tensions énormes entre ceux qui favorisent la poursuite de la recherche comme elle est et les tenants d'une position où la réflexion éthique occupe une place plus importante. Convaincus qu'ils faisaient de la bonne science et que les problèmes rapportés lors du jugement de Nuremberg s'appuyaient sur des inten. tions perverses qu'ils ne croyaient pas leur, la majorité des scientifiques américains de l'époque oubliaient que, juste avant la guerre, ils travaillaient en étroite collaboration avec les scientifiques alle. mands et que chez eux aussi, certaines pratiques eugéniques, par exemple, avaient été promues. S'inscrivant dans un courant d'éveil aux répercussions sociales des développements scientifiques, courant marqué entre autres par les questions portant sur l'utilisation de l'atome et sur les effets environnementaux, le texte de Beecher sera reconnu comme un autre moment significatif du développement de l'éthique de la recherche.

Nul doute que la bioéthique puise fortement dans l'évolution et les problèmes qui ont marqué la recherche, l'histoire que nous venons trop rapidement de brosser le démontre. Dès la période initiale, elle présente, comme le dit Jonsen, des «signes de la nature 
réelle de la science biomédicale moderne : l'innovation, la nécessité de produire et l'originalité 20 ».

\section{Espoirs et tensions de la période initiale sur l'éthique de la recherche}

Un examen de la littérature de la période initiale nous a conduit à retenir trois textes qui, à notre avis, marquent de façon importante la première décennie du courant bioéthique et formulent les espoirs des fondateurs en ce qui concerne l'éthique de la recherche. Ce sont des textes de Hans Jonas, Van Ressler Potter et le Rapport Belmont.

Publié en 1969 sous le titre Philosophical Reflections on Experimenting With Human Subjects ${ }^{21}$, ce texte de Jonas eut, dès ses débuts, une importance considérable. En témoigne sa fréquente reproduction, en totalité ou dans une version écourtée, dans d'autres œuvres ${ }^{22}$. J'en présente d'abord les éléments principaux de la version écourtée. Qualifiant la recherche médicale comme la plus légitime, la recherche psychologique comme la plus douteuse et la recherche biologique comme la plus dangereuse, le texte, dans sa version écourtée, poursuit en soulignant la polarité qui s'inscrit entre les droits, les besoins et les intérêts de l'individu et ceux de la société. Dans une section portant sur le santé comme bien public, Jonas poursuit en présentant les conditions d'une réalisation digne de

A.R. JONSEN et R.A. PEARLMAN, The Birth of Bioethics, p. S4.

21 H. JONAS, «Philosophical Reflections on Experimenting With Human Subjects", dans P.A. FREUND (éd.), Experimentation with Human Subjects. New-York, George Braziller, 1969, pp. 1-31.

22 Ce texte se retrouve entre autres dans T.L. BEAUCHAMP et W. LE ROY, Contemporary Issues in Bioethics. Belmont, CA, Woodsworth Publishing Co., 1978, 612 p.; CAHIERS DE BIOÉTHIQUE, Médecine et expérimentation, no 4, Québec, Les Presses de l'Université Laval, 1982, 461 p.; S. GOROVITZ et al. (éd.), Moral Problems in Medicine. Englewood Cliffs, New-Jersey, Prentice-Hall, 1976, 552 p.; G.H. KIEFFER, Bioethics : A Textbook of Issues; T.A. MAPPES et J.S. ZEMBATY, Biomedical Ethics, NewYork, McGraw-Hill Book Co., 1981, 587 p.; S.J. Reiser, DYCK, A.J. DYCK et W.J. CURRAN, Ethics in Medicine. Historical Perspectives and Contemporary Concerns. Cambridge, MA, The M.I.T. Press, 1977, 679 p. 
l'expérimentation. Selon lui, l'utilisation du vocabulaire s'avère alors significative pour déceler la transformation possible des sujets humains en simples objets de recherche. La protection à leur accorder s'enracine dans le droit, la justice et la liberté et tout moyen doit être pris pour s'assurer qu'il y ait protection, surtout en cette époque où, selon Jonas, nous souffrons du syndrôme de la-recherche-avecdes-sujets-humains-perçue-comme-un-intérêt-social. Dans son optique, nos descendants ont droit à une planète qui aura été protégée du pillage mais n'ont pas le même droit à des cures miracles. Il poursuit ensuite en spécifiant les conditions de recrutement des sujets et propose une règle de sélection s'opposant à une perspective utilitariste. Ce faisant, il est conscient que son modèle restreindra la rapidité à laquelle la recherche pourra se développer. Il conclura donc en soulignant qu'une lente progression dans le combat contre la maladie ne constitue pas une menace aussi dangereuse pour la société que l'érosion et la perte des valeurs morales à laquelle une progression plus rapide pourrait conduire.

La version écourtée supprime cependant des passages particuliè rement intéressants qui méritent de retenir l'attention. Voici donc quelques passages du texte original. La première section absente présente la recherche médicale en tant qu'émule de la recherche en sciences naturelles avec cette différence qu'en se basant sur des sujets humains, la première perd l'innocence de la quête de connaissance que possède la seconde et favorise alors l'avènement des ques tions de conscience. Dans une deuxième section absente qui représente environ $20 \%$ du document, la question du consentement, les thèmes du sacrifice et du contrat social sont abordés. Enfin, les autres blocs enlevés qui sont tout aussi importants quant au nombre de pages, touchent la loi morale, à nouveau le consentement et le recrutement, l'expérimentation sur des patients, la limite ténue qui existe entre traitement et expérimentation et, enfin, la mort. Ces éléments soulignent la responsabilité sociale de la recherche tout en se remémorant et en tirant leçon de l'expérience difficile des camps de concentration nazis.

Avec le texte de Jonas, les premières bases philosophiques de la bioéthique moderne sont posées. Il constitue d'autant plus un jalon important qu'à cette époque, la présence des philosophes dans le mouvement est très restreinte et la perspective pragmatiste prend 
forme. La proposition d'une conduite scientifique responsable qui devient manifeste à la lecture du texte, conduira Jonas à la production d'une de ses œuvres importante The Imperative of Responsibility ${ }^{23}$.

Le deuxième texte, celui de Van Ressler Potter intitulé Bioethics for Whom ${ }^{24}$, met lui aussi l'accent sur la responsabilité en s'adressant particulièrement aux scientifiques. Texte important puisqu'il présente l'essentiel d'une des deux approches bioéthiques ouvertes au développement ${ }^{25}$, Potter y rejette tout accroissement des contrôles externes des scientifiques sur la base de son opposition aux contrats de recherche comme mode de support des professeurs. Il y invite les scientifiques et leurs organisations à passer à l'action en s'autorégulant et en établissant leurs priorités. Inscrivant la responsabilité dans le respect propre de la liberté académique, il favorise l'établissement de lignes directrices déterminées dans le cadre d'un dialogue entre scientifiques et non-scientifiques. Ces lignes directrices devraient nécessairement inclure la divulgation de tout bénéfice, des risques encourus et l'établissement d'une discussion ouverte concernant les situations considérées comme embarrassantes.

Étant donnée sa signification historique majeure, nous ne croyons pas nécessaire de souligner l'importance du troisième texte, le Rapport Belmont. Certains auteurs pourraient cependant critiquer le fait que nous le considérions dans la période initiale de la bioé thique. À notre avis, il se situe à la frontière de deux périodes si nous prenons en considération le fait que les travaux de la commission

23 H. JONAS, The Imperative of Responsibility. In Search of an Ethics for the Technological Age. Chicago, The University of Chicago Press, 1984, $255 \mathrm{p}$.

24 R. VAN POTTER, "Bioethics for Whom", Annals of the New-York Academy of Sciences, vol. 196, 1972, p. 200-205.

25 Les deux approches qui se sont manifestées en bioéthique au cours des années 1970 peuvent être représentées par deux auteurs. D'un côté, l'approche d'André Hellegers se concentre essentiellement sur l'évolution de la science en milieu médical. De l'autre, Van Ressler Potter propose une approche de type holistique considérant l'effet de l'évolution de la science sur la nature et la société. 
aient débuté lors de la période initiale, que les ébauches aient été écrites en 1975 et 1976 tandis que le document final a été publié en 1978. La majeure partie de la préparation du rapport ayant eu lieu lors de la prériode initiale, nous l'incluons donc dans celle-ci. De plus, il est le texte fondateur de l'éthique de la recherche.

Ayant pour titre exact Le rapport Belmont: Principes d'éthique et lignes directrices pour la recherche faisant appel à des sujest humains ${ }^{26}$, ce document porte, comme les deux autres, sur l'aspect responsabilité du travail, dans ce cas, du chercheur auquel il veut donner des outils pour une pratique éthique de la recherche avec des sujets humains. Situant le document dans la perspective des bénéfices sociaux engendrés par la recherche, les auteurs, dans un premier temps, tracent une ligne de démarcation entre la pratique et la recherche en les plaçant dans le contexte des abus de certaines expériences biomédicales. La pratique y est présentée comme l'intervention visant à améliorer, avec une espérance raisonnable de succès, le bien-être du patient. En ce qui concerne la recherche, les auteurs la décrivent comme une activité visant à vérifier des hypothèses, tirer des conclusions et permettre ainsi l'avancement des connaissances. Fait important, dans les sections suivantes, le rapport présente les trois principes qui constitueront la base de ce qui deviendra le courant majeur de la bioéthique. Premièrement, selon le principe du respect des personnes, les individus doivent être traités comme des agents autonomes tout en tenant compte que les personnes ayant une autonomie diminuée ont droit à être protégées. Deuxièmement, selon le rapport, le principe de bienfaisance ajoute que les personnes sont traitées éthiquement non seulement lorsque leurs décisions sont respectées et qu'elles sont protégées contre les dangers mais aussi lorsqu'un effort est fait pour assurer leur bien-être. Enfin, le principe de justice s'intéresse à la question des bénéficiaires de la recherche et des personnes qui en portent le fardeau.

NATIONAL COMMISSION FOR THE PROTECTION OF HUMAN SUBJECTS OF BIOMEDICAL AND BEHAVIORAL RESEARCH (USA), The Belmont Report. Ethical Principles and Guidelines for the Protection of Human Subjects of Research. Department of Health, Education and Welfare, no (OS) 78-0012, 1978. 
Au cours des années qui suivront, d'autres publications attireront l'attention sur les problèmes éthiques entourant la recherche. Ces textes comprendront, mais aussi dépasseront, les limites des sciences biomédicales et le traitement des sujets humains 27 . Cependant, aucun n'aura l'impact du texte de Beecher. De leur côté, les organismes scientifiques et pouvoirs gouvernementaux continueront à proposer des cadres de référence, des normes, des règlements et des législations. Peu à peu toutefois, la visibilité de l'éthique de la recherche s'estompera au profit de l'accroissement de l'activité en éthique médicale et en éthique clinique. Un regard sur les publications des années 1980 montre clairement ce déplacement.

Les sujets abordés portent de plus en plus sur les situations cliniques et l'éthique de la recherche en vient à occuper une place moins importante quant à la position et au nombre de pages qui lui sont accordées dans les publications. Le texte de Jonas glisse dans l'oubli. Le courant holiste, favorisé par Van Potter, perd du terrain pour se retrouver dans l'ombre de l'approche nettement biomédicale de André Hellegers ${ }^{28}$. Quant au Rapport Belmont, Beauchamp et Childress en reprendront les principes et les développeront en les inscrivant dans un contexte essentiellement clinique délaissant ainsi sa dimension originelle liée à l'éthique de la recherche ${ }^{29}$.

Dans un article intitulé Whatever Happened to Research Ethics, Baruch Brody indique que :

Au cours des dix ou quinze dernières années, la communauté bioéthique a porté peu d'attention à une importante série de questions qui avaient été discutées dans la communauté de recherche comme dans la communauté politico-juridique. Ayant

27 Voir à ce sujet W. BRODE et N. WADE, Betrayers of the Truth. Fraud and Deceit in the Halls of Science. New York, Simon and Schuster, Inc., 1982, 256 p.; A. KOHN, False Prophets. New York, Basil Blackwell Inc., $1986,226 \mathrm{p}$.

28 H. DOUCET, Au pays de la bioéthique, p. 39. Il est à noter que la paternité du terme "bioéthique " est accordée tant à Hellegers qu'à Potter. 29 T.L. BEAUChAmP et J.F. CHILDRESS, Principles of Biomedical Ethics, $314 \mathrm{p}$. 
réussi à défendre un cadre général visant à protéger les sujets humains dans la recherche aux États-Unis comme ailleurs, elle s'est tournée vers d'autres horizons, ignorant virtuellement l'éthique de la recherche. Bien sûr, certains secteurs de la recherche attirent toujours l'attention (spécialement la recherche génétique, fœtale et reproductive) et des périodiques ainsi que des individus... ont continué à s'intéresser à ces sujets. Mais des questions importantes et fondamentales en éthique de la recherche ne reçoivent pas l'attention qu'elles devraient de la part des bioéthiciens... Une liste partielle (de ces sujets) inclut la fraude et la mauvaise conduite, les conflits d'intérêts, la propriété intellectuelle des résultats de recherche, la méthode des essais cliniques et l'approbation des résultats de recherche ${ }^{30}$.

La situation est-elle si alarmante? De fondatrice de la bioéthique, l'éthique de la recherche en est-elle devenue le parent pauvre? Qu'est-il advenu du rêve de ses fondateurs?

\section{Espoirs et tensions en cette fin de $\mathrm{XX}^{\mathrm{e}}$ siècle}

En réponse aux commentaires de Brody, Harold Vanderpool indique que pour la période de 1994-1995, 115 articles et 12 livres ont été publiés dont les sujets concernaient l'éthique et la réglementation de la recherche impliquant des sujets humains 31 . À cela s'ajoutent 24 conférences. À notre avis cependant, tant Brody que Vanderpool ne présentent qu'une facette de la réalité historique de l'éthique de la recherche.

Avec Vanderpool, nous reconnaissons l'étroitesse de l'échantillonage des textes sur lequel l'hypothèse de Brody repose. L'analyse de la période initiale de la bioéthique que nous venons d'effectuer nous

30 B. BRODY, "Whatever Happened to Research Ethics", dans R.A. CARSON et C.B. BURNS (éd.), Philosophy of Medicine and Bioethics. A Twenty-Year Retrospective and Critical Appraisal. Boston, Kluwer Academic Publishers, 1997, pp. 275-276.

31 H.Y. VANDERPOOL, "What's Happening in Research Ethics? Commentary on Brody", dans R.A. CARSON et C.B. BURNS (éd.), Philosophy of Medicine and Bioethics, p. 291. 
porte cependant à avancer que la représentation brossée par Brody s'avère une juste description de l'intérêt de la majorité des bioéthiciens pour l'éthique de la recherche au cours de la deuxième période du mouvement débutant au milieu des années 1970 et se terminant avec les années $1980^{32}$.

Au début des années 1990, l'intérêt public pour l'éthique de la recherche se raviva. En 1989, puis, à nouveau, après six ans d'activités autour du sujet, en 1995, la National Academy of Sciences, la National Academy of Engineering et l'Institute of Medicine des ÉtatsUnis publièrent On Being A Scientist: Responsible Conduct In Research ${ }^{33}$. Au Canada, plusieurs conférences portant sur l'intégrité de la recherche furent tenues. En 1994, les trois conseils subventionnaires canadiens énoncèrent une politique sur l'intégrité dans la recherche et les travaux d'érudition à laquelle les universités canadiennes durent adhérer ${ }^{34}$. Certains événements comme l'affaire Fabrikant (1992) et, quelques années plus tard, l'affaire Poisson, secouèrent le public. Une pression sociale commença à s'exercer sur les milieux de recherche. Les projets de recherche de certains conseils subventionnaires furent scrutés à la loupe pour en déterminer la pertinence économique pour la société canadienne. Les travaux des trois conseils subventionnaires canadiens débutèrent dans l'optique d'élaborer initialement un guide d'éthique de la recherche impliquant des sujets humains. La situation n'était pas unique à l'Amérique du Nord. Dans cette effervescence du milieu de la décennie 1990, il n'est donc pas surprenant que Vanderpool ait recensé un si grand nombre de publications et de conférences.

H. DOUCET, Au pays de la bioéthique, p. 43.

33 COMMITTEE ON SCIENCE, ENGINEERING AND PUBLIC POLICY, On Being A Scientist : Responsible Conduct In Research. Washington, D.C., National Academy Press, 1995, 33 p.

34 CONSEIL DE RECHERCHES MÉDICALES DU CANADA, CONSEIL DE RECHERCHES EN SCIENCES NATURELLES ET GÉNIE DU CANADA ET CONSEIL DE RECHERCHES EN SCIENCES HUMAINES DU CANADA, L'intégrité dans la recherche et les travaux d'érudition, Ottawa, janvier 1994, s.e., 4 p. 
En 1969, Jonas centrait son propos sur la recherche médicale tout en soulignant les dangers de la recherche psychologique et de la recherche biologique. Ces secteurs d'activité se sont développés ouvrant la porte à de nouvelles questions éthiques. Qui plus est, des scientifiques d'autres secteurs de sciences naturelles, sociales et humaines ont perçu la nécessité d'un comportement responsable et d'une vigilence éthique. Le traitement possible des sujets humains comme objets nous semble maintenant mieux encadré mais cet encadrement est souvent imposé par le gouvernement, l'effort d'autorégulation des scientifiques souhaité par Jonas ne s'étant pas montré adéquat. Il faut cependant considérer que les conditions d'une telle autorégulation décrite par Potter ne se sont pas matérialisées.

Dans un contexte de coupures budgétaires, les universités se sont tournées de plus en plus vers les contrats de recherche puis vers la commandite. L'avenue des partenariats fut prise allègrement sans bien en mesurer les répercussions éthiques. En considérant l'optique de Potter, il n'est donc pas surprenant que des contrôles sociaux addi tionnels s'exercent. Qu'il s'agisse de recrutement des sujets, de divulgation des bénéfices ou des risques possibles de la recherche, des outils existent mais des questions demeurent. Dans son texte, Jonas disait qu'il valait mieux un combat lent contre la maladie que l'éro. sion et la perte des valeurs morales. Avons-nous suffisamment réfléchi sur les répercussions pratiques et éthiques de ces avenues empruntées par notre société? Le combat contre la maladie est bien engagé mais la question reste et restera toujours ouverte, comme en veilleuse.

\section{Conclusion}

Il y a trente ans, la bioéthique naissait aux États-Unis, en réponse aux questionnements scientifiques et sociaux émergeant des avancées techno-scientifiques. Ce nouveau mouvement social fut plus particulièrement appelé par une activité de recherche en pleine 
ébullition, activité qui constitue un « des trois lieux d'intersection de l'éthique avec la technoscience ${ }^{35} \%$.

Il y a trente ans, des médecins, des théologiens, des chercheurs et quelques philosophes ont commencé, d'une manière bien pragmatique, à discuter et à chercher en collaboration des réponses à des questions auxquelles ils étaient quotidiennement confrontés. C'est dans une perspective de responsabilité et de protection des sujets humains qu'ils ont inscrit leur démarche.

Aujourd'hui, dans le cadre d'une entreprise de recherche toujours plus active, avec la mutiplication des équipes multidisciplinaires et des travaux multicentres, avec le développement des contrats de recherches et des partenariats entre divers secteurs de la société, la bioéthique est encore et toujours invitée à répondre à des questions très pratiques. Saura-t-elle relever le défi? Elle a pris le chemin des régulations imposées par les autorités gouvernementales. Les questions fondamentales soulevées par Hans Jonas, Van R. Potter et le rapport Belmont ont été quelque peu occultées. Peut-être est-il temps de revenir aux questions du sens de la recherche biomédicale, du contexte de sa réalisation et de ses implications pour l'humanité d'aujourd'hui et de demain.

35 G. HOTTOIS, «La Bioéthique : définitions, problèmes et méthodologie », dans Réseaux 53-54 (1987-1988) 18. 